University of Nebraska - Lincoln

DigitalCommons@University of Nebraska - Lincoln

\title{
Validation of monoclonal antibody F99/97.6.1 for immunohistochemical staining of brain and tonsil in mule deer (Odocoileus hemionus) with chronic wasting disease
}

\author{
T. R. Spraker \\ Colorado State University - Fort Collins \\ Katherine I. O'Rourke \\ U.S. Department of Agriculture, katherine.orourke@ars.usda.gov
}

A. Balachandran

Animal Disease Research Inspection

R. R. Zink

Colorado State University - Fort Collins

B. A. Cummings

Colorado State University - Fort Collins

See next page for additional authors

Follow this and additional works at: https://digitalcommons.unl.edu/zoonoticspub

Part of the Veterinary Infectious Diseases Commons

Spraker, T. R.; O'Rourke, Katherine I.; Balachandran, A.; Zink, R. R.; Cummings, B. A.; Miller, M. W.; and Powers, B. E., "Validation of monoclonal antibody F99/97.6.1 for immunohistochemical staining of brain and tonsil in mule deer (Odocoileus hemionus) with chronic wasting disease" (2002). Other Publications in Zoonotics and Wildlife Disease. 133.

https://digitalcommons.unl.edu/zoonoticspub/133

This Article is brought to you for free and open access by the Wildlife Disease and Zoonotics at DigitalCommons@University of Nebraska - Lincoln. It has been accepted for inclusion in Other Publications in Zoonotics and Wildlife Disease by an authorized administrator of DigitalCommons@University of Nebraska Lincoln. 


\section{Authors}

T. R. Spraker, Katherine I. O'Rourke, A. Balachandran, R. R. Zink, B. A. Cummings, M. W. Miller, and B. E. Powers 


\title{
Validation of monoclonal antibody F99/97.6.1 for immunohistochemical staining of brain and tonsil in mule deer (Odocoileus hemionus) with chronic wasting disease
}

\author{
T. R. Spraker, K. I. O’Rourke, A. Balachandran, R. R. Zink, B. A. Cummings, M. W. Miller, \\ B. E. Powers
}

\begin{abstract}
A new monoclonal antibody (MAb), F99/97.6.1, that has been used to demonstrate scrapieassociated prion protein $\mathrm{PrP}^{\mathrm{Sc}}$ in brain and lymphoid tissues of domestic sheep with scrapie was used in an immunohistochemistry assay for diagnosis of chronic wasting disease (CWD) in mule deer (Odocoileus hemionus). The MAb F99/97.6.1 immunohistochemistry assay was evaluated in brain and tonsil tissue from 100 mule deer that had spongiform encephalopathy compatible with CWD and from 1,050 mule deer outside the CWD-endemic area. This MAb demonstrated abnormal protease-resistant prion protein (PrPres) in brains of all of the 100 mule deer and in 99 of the 100 tonsil samples. No immunostaining was seen in samples collected from deer outside the endemic area. MAb F99/97.6.1 demonstrated excellent properties for detection of PrPres in fresh, frozen, or mildly to moderately autolytic samples of brain and tonsil. This immunohistochemistry assay is a sensitive, specific, readily standardized diagnostic test for CWD in deer.
\end{abstract}

Chronic wasting disease (CWD) is one of several transmissible spongiform encephalopathies affecting animals and is the only one found in free-ranging mule deer (Odocoileus hemionus), white-tailed deer (O. virginianus), and Rocky Mountain elk (Cervus elaphus nelsoni). ${ }^{14}$ CWD is most reliably diagnosed in fresh fixed sections of brain stem, especially the dorsal motor nucleus of the vagus nerve (DMNV), where spongiform encephalopathy is found. However, fresh brain specimens are usually not available, especially in field surveys of cervids for CWD. Several slightly different immunohistochemistry (IHC) techniques have been developed for detection of protease-resistant prion protein $\left(\mathrm{PrP}^{\mathrm{res}}\right)$ in elk and deer. ${ }^{2,3,10-14,16}$ Positive IHC results have been interpreted as evidence that a scrapieassociated prion protein or an antigenically similar PrPres is present in lymphoid tissues and brain of captive and free-ranging deer and elk. ${ }^{2,3,10-14}$ Therefore, detection of PrPres in tissues would help to confirm CWD in questionable cases. The objective of this investigation was to evaluate the utility of monoclonal antibody (MAb)-based IHC assay for detection of $\mathrm{PrP}^{\mathrm{res}}$ in mule deer with CWD. This assay can be used in antemortem testing of experimentally inoculated an-

From the Department of Pathology, College of Veterinary Medicine, Colorado State University, Fort Collins, CO 80523 (Spraker, Zink, Cummings, Powers), the Animal Disease Research Unit, ARS/ USDA, 3003 ADBF, Pullman, WA 99164 (O’Rourke), Animal Disease Research Inspection, CFIA, 3851 Fallowfield Road, Nepean, Ontario K2G 5HP, Canada (Balachandran), and the Colorado Division of Wildlife, 317 West Prospect Road, Fort Collins, CO 80523 (Miller).

Received for publication July 5, 2001. imals in the preclinical stages of the disease and in antemortem and postmortem sampling of free-ranging animals to estimate CWD prevalence.

\section{Materials and methods}

This study included 100 mule deer that had a spongiform encephalopathy of the brain histologically compatible with CWD. Sections of palatine tonsil and multiple sections of brain, with 1 section always from the medulla at the level of the obex and including the DMNV, were preserved in $10 \%$ neutral buffered formalin and embedded in paraffin. Duplicate sections were cut at $5 \mu \mathrm{m}$ and mounted on positively charged glass slides. One slide was stained with hematoxylin and eosin (HE), ${ }^{7}$ and the other was assayed by MAb F99/97.6.1 IHC (MAb99-IHC). MAb F99/97.6.1 ${ }^{\mathrm{a}}$ reacts with a conserved epitope (residues QYQRES) on the prion protein of mule deer, Rocky Mountain elk, domestic sheep, and cattle. ${ }^{10}$ MAbs specific for scrapie-associated prion protein $\mathrm{PrP}^{\mathrm{Sc}}$ in formalin-fixed tissues have not been described. Formalin fixation and formic acid treament-AP. ${ }^{1}$ reduces or eliminates the availability of the epitope for MAb F99/97.6.1 in cervid brain and lymphoid tissues. Antigen retrieval with formic acid ${ }^{6}$ and hydrated autoclaving 5 selectively unmasks the epitope on $\mathrm{PrP}^{\mathrm{Sc}}$ in fixed tissues.

The CWD-positive mule deer in this study had been diagnosed by examination of HE-stained slides with confirmation by IHC of brain tissues using MAb F89/160.1.5. ${ }^{11}$ The epitope for MAb F89/160.1.5 (residues IHFG) on sialoglycoprotein $\left(\mathrm{PrP}^{\mathrm{sen}}\right)$ is eliminated by formalin fixation and formic acid treatment in cervid brain, as evidenced by the failure to detect immunostaining in brain samples from more than 1,000 deer harvested outside the CWD-endemic area. Preliminary trials of antigen retrieval conditions for lymphoid tissue demonstated that the intensity of immunostaining was higher when a modified citrate acid buffer ${ }^{\mathrm{b}}$ was used 


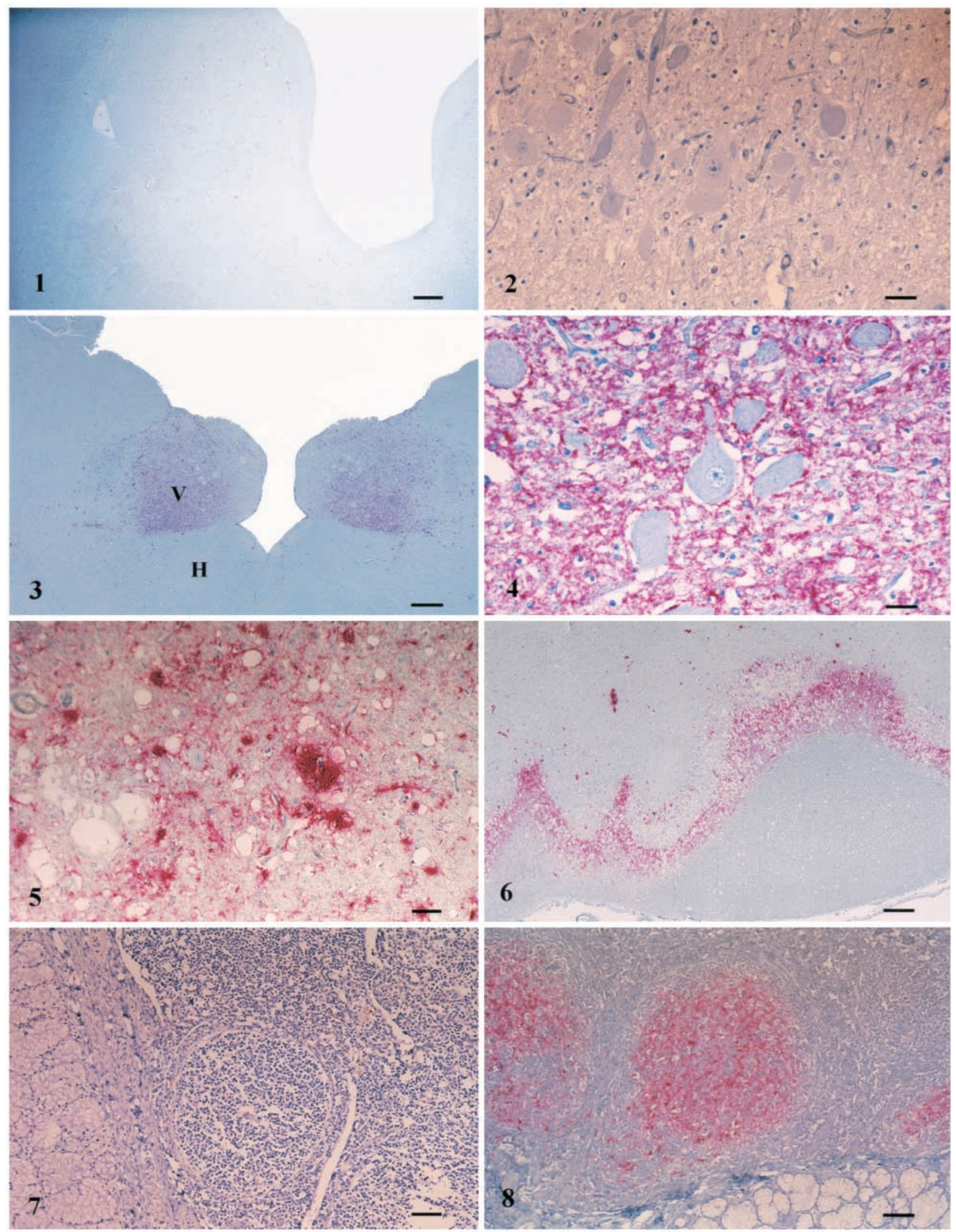

Figure 1. Obex, brain (low power) from a mule deer that does not have chronic wasting disease. Note the absence of any red chromogen staining. Immunohistochemical staining. Streptavidin-alkaline phosphatase method. Hematoxylin/bluing counterstain. Bar $=650 \mu \mathrm{m}$. 
in the hydrated autoclaving step. However, approximately $3-$ $5 \%$ of tonsil samples collected from mule deer outside the CWD-endemic area showed variable patterns of immunostaining with MAb F89/160.1.5 after citrate buffer hydrated autoclaving. This immunostaining probably represents detection of the normal cellular PrPsen by MAb F89/160.1.5 in some cervid lymphoid tissues prepared by this method. This staining was not detectable using MAb F99/97.6.1 under these staining conditions. Therefore, only MAb F99/97.6.1 was used in the larger study.

Samples of mule deer brain and tonsil were mounted on the same slide. Brain samples were stained with HE, and replicate slides were immunostained with MAb99-IHC as follows. Slides were dried in a $65 \mathrm{C}$ oven overnight, deparaffinized, rehydrated, and immersed in $88 \%$ formic acid for $30 \mathrm{~min}$. The slides were then rinsed in deionized water to remove residual formic acid. The slides were next autoclaved in a citrate buffer $(\mathrm{pH} 6.1)^{\mathrm{b}}$ for $20 \mathrm{~min}$ at $121 \mathrm{C}$, cooled to room temperature, rinsed with deionized water, and placed in a Tris buffer. ${ }^{\mathrm{c}}$ MAb99-IHC was performed with a NexES automated immunostainer ${ }^{\mathrm{c}}$ at $37 \mathrm{C}$ using an alkaline phosphatase red kit.c Primary MAb F99/97.6.1 was diluted to an $\mathrm{IgG}$ concentration of $10 \mu \mathrm{g} / \mathrm{ml}$ in diluent ${ }^{\mathrm{c}}$ and placed in the refillable dispenser provided with the immunostainer. The primary antibody, MAb F99/97.6.1, was applied to the slides for $32 \mathrm{~min}$. Slides were counterstained on the immunostainer with hematoxylinc for $4 \mathrm{~min}$ and with bluing ${ }^{\mathrm{c}}$ for another 4 min.

Criteria used for histologic diagnosis of CWD in HEstained tissues included intraneuronal vacuolation, microcavitation of gray matter, neuronal degeneration and loss, and mild astrocytosis in the brain stem. ${ }^{14,16-18}$ Criteria for IHC diagnosis of CWD on MAb99-IHC-treated slides included detection of granular red chromogen deposits in brain or tonsil. The palatine tonsil was considered positive in mule deer if any follicle contained cells with positive MAb99-IHC staining. Negative palatine tonsils had no staining in cells of lymphoid follicles. A typical section of tonsil contained approximately 100-200 follicles.
Negative controls for this study included brain and tonsil from 300 mule deer collected from areas within Colorado but outside of the areas endemic for CWD. ${ }^{9}$ In these mule deer, a single section of obex and tonsil were stained with HE and MAb99-IHC. Free-ranging mule deer $(n=750)$ collected from Montana, where CWD has not been found in the wild, were also used as negative controls.

\section{Results}

Spongiform encephalopathy and PrPres was not observed in the brain stem of any of the negative control mule deer examined in this study (Figs. 1, 2).

PrPres was demonstrated by MAb99-IHC in the brain sections of all of the mule deer that had spongiform encephalopathy. PrPres in brain was characterized by coarse granular staining by the red chromogen. The staining was located on the periphery around neurons and in the neuropil, often in plaques adjacent to or near glial cells (Figs. 3, 4). There were also plaques with red chromogen stain randomly scattered throughout the neuropil, especially in the thalamus (Fig. 5). An abundance of PrPres was found in the olfactory cortex (Fig. 6).

Ninety-nine of the 100 mule deer had extensive staining within follicles of the palatine tonsil. Staining in the tonsils was in the germinal centers of the lymphoid follicles, but the $\mathrm{PrP}^{\mathrm{Sc}}$-positive cell type cannot be distinguished microscopically because of the extensive cytoplasmic processes of the follicular dendritic cells interdigitating with lymphocytes and macrophages. One deer with extensive spongiform encephalopathy and positive MAb99-IHC staining in the brain did not have any tonsillar staining in lymphoid follicles. The tonsil and brain of this mule deer were also immunostained with MAb F89/160.5.1, and this assay

Figure 2. Obex, brain (high power) from a mule deer that does not have chronic wasting disease. Note the absence of any red chromogen staining. Immunohistochemical staining. Streptavidin-alkaline phosphatase method. Hematoxylin/bluing counterstain. Bar $=30 \mu \mathrm{m}$.

Figure 3. Obex, brain (low power) from a mule deer with chronic wasting disease. Note the abundant red chromogen filling the dorsal motor nuclei of the vagus (V) and the lack of staining in the hypoglossal nuclei (H). Immunohistochemical staining. Streptavidin-alkaline phosphatase method. Hematoxylin/bluing counterstain. Bar $=650 \mu \mathrm{m}$.

Figure 4. Obex, brain (high power) from a mule deer with chronic wasting disease. Note the abundant red chromogen associated with neurons and in the adjacent neuropil of the dorsal motor nuclei of the vagus. Immunohistochemical staining. Streptavidin-alkaline phosphatase method. Hematoxylin/bluing counterstain. Bar $=70 \mu \mathrm{m}$.

Figure 5. Thalamus, brain from a mule deer with chronic wasting disease. Note the red chromogen in the neuropil and the plaques of PrPres. Immunohistochemical staining. Streptavidin-alkaline phosphatase method. Hematoxylin/bluing counterstain. Bar $=30 \mu \mathrm{m}$.

Figure 6. Olfactory cortex, brain from a mule deer with chronic wasting disease. Note the distinct area of abundant red chromogen in the olfactory cortex. Immunohistochemical staining. Streptavidin-alkaline phosphatase method. Hematoxylin/bluing counterstain. Bar = $330 \mu \mathrm{m}$.

Figure 7. Palatine tonsil lymphoid follicles from a mule deer that does not have chronic wasting disease. Note the lack of red chromogen deposition in the lymphoid follicle. Immunohistochemical staining. Streptavidin-alkaline phosphatase method. Hematoxylin/bluing counterstain. Bar $=240 \mu \mathrm{m}$.

Figure 8. Palatine tonsil lymphoid follicles from a mule deer with chronic wasting disease. Note the abundant red chromogen deposition in the lymphoid follicle. Immunohistochemical staining. Streptavidin-alkaline phosphatase method. Hematoxylin/bluing counterstain. Bar $=240 \mu \mathrm{m}$ 
also demonstrated strong staining in the brain but not in the lymphoid follicles of the tonsil.

A negative palatine tonsil had no staining in lymphoid follicles (Fig. 7). PrPres in the palatine tonsil was characterized by a coarse granular, bright red material in lymphoid follicles (Fig. 8). In a typical positive palatine tonsil section, $\geq 50 \%$ of the follicles were filled with bright red chromogen.

Freeze artifacts in brain and tonsil varied from minor to marked tissue clefting. Autolysis varied from mild clear spaces around neurons, lymphoid cells, and vasculature to tissue architecture disruption with clear spaces and loss of staining with hematoxylin. Regardless of the degree of freeze artifact or autolysis, immunostaining was not affected.

\section{Discussion}

CWD is a fatal neurologic disease of free-ranging mule deer and white-tailed deer in northcentral Colorado, southern Wyoming, western Nebraska, and a small region of Saskachewan, Canada. CWD is a member of the prion family of disorders, a novel group of transmissible spongiform encephalopathies (TSEs) characterized by accumulation of a protease-resistant isoform (PrPres) of a normal host sialoglycoprotein $\left(\mathrm{PrP}^{\mathrm{sen}}\right)$ in the brain of affected individuals. The TSEs of domestic livestock include ovine scrapie, bovine spongiform encephalopathy, and a CWD of gameraised elk in the USA, Canada, and Korea and of freeranging elk in the mule deer CWD-endemic area.

The TSEs of ruminant herbivores are characterized by spongiform lesions in the central nervous system of clinically affected animals. The lesions are associated with PrPres detectable by IHC of formalin-fixed tissue. PrPres accumulation precedes the appearance of microscopic lesions in the brain of sheep ${ }^{8}$ and elk $^{12}$ and is therefore a reliable marker for TSE in sheep or elk during the preclinical stage of the disease. PrPres can also be detected in tonsil of sheep with TSE months or years before accumulation in the brain can be detected. ${ }^{15}$ Early lymphoid accumulation of PrPres is seen in experimentally infected mule deer ${ }^{13}$ and naturally exposed mule deer in the CWD-endemic area. Therefore, IHC assays on tonsil and other lymphoid tissues ${ }^{10}$ of sheep and mule deer are useful preclinical antemortem and postmortem diagnostic tests for ovine scrapie. In this study, an assay for detection of PrPres in brain and tonsil tissue from mule deer with naturally occurring CWD was evaluated. A sensitive, specific MAb-based IHC assay for identifying preclinically infected deer will be useful in large-scale surveillance studies and in experimental studies on pathogenesis and transmission of CWD in mule deer and related species.

In this study, the use of MAb F99/97.6.1 in an IHC assay of formalin-fixed tissues was examined. MAbbased assays may be more useful than those based on rabbit or goat antisera, which are limited in quantity, specificity, and often in titer. However, use of an MAb with specificity restricted to a single epitope might lead to false-negative findings in outbred species if the antibody binding site is variable. The deduced amino acid sequence of the epitope for MAb F99/97.6.1 is conserved in sheep and cattle, and no variation in the DNA encoding this site has been found in a survey of more than 100 mule deer and white-tailed deer (data not shown).

PrPres has the same primary amino acid sequence as the ubiquitously expressed cellular isoform PrPsen. No commercially available antibody distinguishes between the cellular isoform and the disease-specific isoform in IHC. Specificity for the disease-associated form of the protein in IHC is achieved by treatment of the tissue with formalin and formic acid, which reduces or eliminates the availability of the epitope on $\mathrm{PrP}^{\mathrm{sen}} 1$ followed by antigen retrieval with formic acid ${ }^{6}$ and hydrated autoclaving. ${ }^{5}$ The formalin and formic acid sensitivity of epitopes on PrP isoforms varies with species and with tissue type. Formic acid pretreatment of formalin-fixed lymphoid tissue from sheep before processing and embedment (in addition to treatment of hydrated sections on slides before autoclaving) is required for specificity in an IHC assay using a cocktail of MAbs F89/160.1.5 and F99/97.6.1, although pretreatment of brain before the processing stage does not appear necessary. Likewise, immunostaining with MAb F89/160.1.5 in this study was highly specific in deer brain but resulted in false-positive reactions in some samples of deer tonsil, probably because of detection of the epitope on $\mathrm{PrP}^{\mathrm{sen}}$.

Immunostaining with MAb F99/97.6.1 using the protocol described here is a sensitive and specific test for detecting PrPres in palatine tonsil and brain of mule deer with CWD. This technique detected PrPres in brain of all 100 CWD-positive mule deer in the study and in tonsil of 99 of these deer. One animal showed no detectable tonsillar PrPres in spite of abundant neural deposition. Accumulation of $\mathrm{PrP}^{\mathrm{res}}$ in the central nervous system but not the lymphoid tissues has been noted in a small subset of sheep with ovine scrapie. ${ }^{4,10,15}$ Tissue tropism may be affected by dose, route of exposure, agent strain differences, host genetics, or the level of $\mathrm{PrP}^{\mathrm{sen}}$ expression in host lymphoid and neural tissues. Postmortem sampling of paired samples of brain and tonsil will provide the most sensitive indicator of CWD in mule deer. If tonsil is the only tissue sampled in antemortem studies, the low percentage of mule deer with no detectable lymphoid PrPres immunostaining should not affect large-scale 
prevalence studies but may be a consideration in smaller experimental trials.

Freezing and autolysis did not seem to play a role in affecting the IHC results for these tissues. The value of this technique is especially evident in large surveys of cervids for CWD. In the last 6-7 years, extensive surveys for the presence of CWD have been done in free-ranging deer and in free-ranging and captive elk. ${ }^{9}$ Often, several postmortem days have elapsed by the time the samples are processed, making the diagnosis of CWD difficult or impossible with HE staining. The use of IHC has greatly facilitated the ability to diagnose CWD in these autolytic field specimens.

MAb99-IHC as described here is a specific and sensitive assay for diagnosing CWD in mule deer by detecting $\mathrm{PrP}^{\mathrm{res}}$ in palatine tonsil and brain. The technique will also have value in transmission and pathogenesis studies on this emerging TSE.

\section{Acknowledgements}

We thank Keith Aune for allowing us to use tissues from free-ranging deer collected in Montana for negative controls. We also thank Kate Larsen, Caroline Krumm, Helen Hseih, Sheri Hower, and others for collecting and trimming tissues, Maryanne Tryjan for embedding tissues, and Yang Sun Shin and Frank Aquino for cutting tissues. This project was supported in part by USDA/ARS grant CWU 5348-32000-01500D, the Colorado Division of Wildlife, and the Colorado State University Diagnostic Laboratory, College of Veterinary Medicine, Fort Collins, CO.

\section{Sources and manufacturers}

a. VMRD, Pullman, WA.

b. Target Retrieval Solution, DAKO, Carpinteria, CA.

c. Ventana Medical Systems, Tucson, AZ.

\section{References}

1. Diedrich JM, Bendheim PE, Kim KS, et al.: 1991, Scrapie-associated protein accumulates in astrocytes during scrapie infection. Proc Natl Acad Sci USA 88:375-379.

2. Guiroy DC, Williams ES, Yanagihara R, et al.: 1991, Immunolocalization of scrapie amyloid (PrP27-30) in chronic wasting disease of Rocky Mountain elk and hybrids of captive mule deer and white-tailed deer. Neurosci Lett 126:195-198.

3. Guiroy DC, Williams ES, Yanagihara R, et al.: 1991, Topographic distribution of scrapie amyloid-immunoreactive plaques in chronic wasting disease in captive mule deer (Odocoileus hemionus hemionus). Acta Neuropathol (Berl) 81:475-478.

4. Hadlow WJ, Race RE, Kennedy RC, et al.: 1979, Natural infection of sheep with scrapie virus. In: Slow transmissible diseases of the nervous system, ed. Prusiner SB, Hadlow WJ, pp. 3-12. Academic Press, New York, NY.

5. Haritani M, Spencer YI, Wells GAH: 1992, Hydrated autoclave pretreatment enhancement of prion protein immunoreactivity in formalin-fixed bovine spongiform encephalopathy-affected brain. Acta Neuropathol (Berl) 87:86-90.

6. Kitamoto T, Ogomori K, Tateishi J, et al.: 1987, Formic acid pretreatment enhances immunostaining of cerebral and systemic amyloids. Lab Invest 57:230-236.

7. Luna LG: 1992, Histopathologic methods and color atlas of special stains and tissues artifacts. Johnson Printers, Downers Grove, IL.

8. Miller JM, Jenny AL, Taylor WD, et al.: 1994, Detection of prion protein in formalin-fixed brain by hydrated autoclaving immunohistochemistry for the diagnosis of scrapie in sheep. $J$ Vet Diagn Invest 6:366-368.

9. Miller MW, Williams ES, McCarty CW, et al.: 2000, Epizootiology of chronic wasting disease in free-ranging cervids in Colorado and Wyoming. J Wildl Dis 36:676-690.

10. O'Rourke KI, Baszler TV, Besser TE, et al.: 2000, Preclinical diagnosis of scrapie by immunohistochemistry of third eyelid lymphoid tissues. J Clin Microbiol 38:3254-3259.

11. O'Rourke KI, Baszler TV, Miller JM, et al.: 1998, Monoclonal antibody F89/160.1.5 defines a conserved epitope on the ruminant prion protein. J Clin Microbiol 36:1750-1755.

12. Peters J, Miller JM, Jenny AL, et al.: 2000, Immunohistochemical diagnosis of chronic wasting disease in preclinically affected elk from a captive herd. J Vet Diagn Invest 12:579-582.

13. Sigurdson CJ, Williams ES, Miller MW, et al.: 1999, Oral transmission and early lymphoid tropism of chronic wasting disease $\mathrm{PrP}^{\mathrm{res}}$ in mule deer fawns (Odocoileus hemionus). J Gen Virol 80:2757-2764.

14. Spraker TR, Miller MW, Williams ES, et al.: 1997, Spongiform encephalopathy in free-ranging mule deer (Odocoileus hemion$u s$ ), white-tailed deer (Odocoileus virginianus) and Rocky Mountain elk (Cervus elaphus nelsoni) in north central Colorado. J Wildl Dis 33:1-6.

15. van Keulen LJM, Schreuder BEC, Meloen RH, et al.: 1996, Immunohistochemical detection of prion protein in lymphoid tissues of sheep with natural scrapie. J Clin Microbiol 34:12281231.

16. Williams ES, Young S: 1980, Chronic wasting disease of captive mule deer: a spongiform encephalopathy. J Wildl Dis 16:89-98.

17. Williams ES, Young S: 1982, Spongiform encephalopathy of Rocky Mountain elk. J Wildl Dis 18:465-471.

18. Williams ES, Young S: 1993, Neuropathology of chronic wasting disease of mule deer (Odocoileus hemionus) and elk (Cervus elaphus nelsoni). Vet Pathol 30:36-45. 